\title{
Portrait sociolinguistique de travailleurs étrangers temporaires agricoles au Québec : entre barrières et besoins
}

\author{
Simon Collin \\ Université du Québec à Montréal \\ Olivier Calonne \\ Université du Québec à Montréal
}

\begin{abstract}
Résumé
L'augmentation du nombre de travailleurs étrangers temporaires (TÉT) constitue une tendance marquante des migrations contemporaines. Dans nombre de travaux, les problématiques linguistiques reviennent en filigrane en ceci qu'elles affectent l'expérience migratoire des TÉT. Pourtant, leur situation sociolinguistique n'a pas été étudiée en tant qu'objet. À partir d'un questionnaire et d'entrevues de groupe auprès de 24 TÉT dans une ferme québécoise, cette recherche a pour objectif de mieux comprendre la situation sociolinguistique des TÉT lors de leur séjour au Québec. Les participants rapportent un niveau linguistique limité en espagnol écrit, en anglais et en français. Ils ont peu d'occasions de parler une autre langue que l'espagnol dans leur quotidien. Cette situation s'explique en partie par le rôle ambigu joué par la société hôte. Les participants vivent une situation sociolinguistique caractérisée par des barrières communicationnelles, situation qui hypothèque largement leurs possibilités d'appropriation linguistique du français. Pour finir, quelques résultats esquissent des pistes d'intervention didactique qui permettraient aux TÉT d'avoir accès des cours de français adaptés à leurs besoins sociolinguistiques.
\end{abstract}

\begin{abstract}
An influx of temporary migrant workers represents a growing trend in the contemporary migration landscape. While many studies note that temporary migrant workers are facing linguistic challenges that are impacting their migration experience, few investigations have examined their sociolinguistic reality. This research aims to better understand the sociolinguistic experience of temporary migrant workers working on a farm in Québec. Twenty-four temporary migrant workers completed a questionnaire and participated in focus groups designed to probe their sociolinguistic experiences. The results demonstrate that while the participants' proficiency in written Spanish, English, and French is limited, the host society affords them few opportunities to communicate in any language other than Spanish. Overall, the participants are facing sociolinguistic barriers that prevent them from acquiring French. Implications for designing French language courses to address migrant workers' sociolinguistic needs are discussed.
\end{abstract}




\section{Portrait sociolinguistique de travailleurs étrangers temporaires agricoles au Québec : entre barrières et besoins}

L'augmentation remarquable du nombre de travailleurs étrangers temporaires (désormais, TÉT dans le reste de cet article) constatée à l'échelle mondiale comme au niveau du Canada et du Québec souligne sans conteste l'une des tendances marquantes de l'évolution des migrations contemporaines. Cette augmentation a été encouragée et encadrée par la mise en place de programmes et de statuts migratoires spécifiques. Bien que la littérature scientifique sur les TÉT soit moins fournie que celle relative à d'autres types de migration, il apparait nettement que les problématiques linguistiques reviennent en filigrane dans nombre de travaux qui leur sont consacrés en ceci qu'elles affectent leur expérience migratoire. Pourtant, la situation sociolinguistique des TÉT n'a pas été étudiée en tant qu'objet, c'est ce que propose cet article. À partir d'un questionnaire et d'entrevues de groupe auprès de 24 TÉT travaillant dans une ferme québécoise, cette recherche avait pour objectif de mieux comprendre la situation sociolinguistique des TÉT lors de leur séjour dans les fermes du Québec. Globalement, les compétences linguistiques des participants s'avèrent limitées en espagnol, en anglais et en français selon leur propre représentation. Par ailleurs, ils ont accès à un nombre réduit de situations linguistiques dans leur quotidien et l'espagnol y domine. De son côté, la société hôte joue un rôle ambigu, entre initiatives d'adaptation et indices de marginalisation. Nous en déduisons que les participants vivent principalement une situation sociolinguistique caractérisée par des barrières linguistiques, situation qui hypothèque largement les possibilités d'appropriation linguistique du français par les TÉT, à la différence d'autres catégories de migrants du Québec. Pour finir, nous présentons quelques résultats qui esquissent des pistes d'intervention didactique permettant aux TÉT d'apprendre le français.

\section{Contexte}

Dans cette section, nous commençons par présenter le contexte politique des TÉT au Canada, envisagé comme une source de bénéfices économiques pour les états signataires, mais également comme une source d'inégalités pour les TÉT en regard des autres catégories de migrants. Nous abordons ensuite le contexte social des TÉT au Canada, indiquant la vulnérabilité et la précarité inhérentes à leur statut migratoire, les conditions de travail difficiles, l'isolement géographique et l'exclusion sociale auxquels ils sont exposés. Ces facteurs permettent de mieux situer le contexte sociolinguistique des TÉT, marqué par un nombre limité d'occasions d'interactions avec les populations locales et par l'existence de nombreuses barrières linguistiques dans les fermes et plus encore, à l'extérieur de cellesci. L'ensemble de ces éléments nous conduit enfin à exposer l'objectif de notre étude qui consiste à mieux comprendre la situation sociolinguistique des TÉT lors de leur séjour dans les fermes du Québec.

\section{Contexte politique des TÉT au Canada}

Comprendre la situation actuelle des TÉT implique dans un premier temps de retracer les politiques qui ont contribué à définir leur statut, ainsi que leurs visées. Dans cette perspective, commençons par noter que les politiques des TÉT au Canada s'inscrivent dans une tendance mondiale encourageant la migration temporaire (Soussi, 2013), sur l'idée 
que cette dernière serait bénéfique à toutes les parties impliquées. L'argument développé consiste à dire que « les pays d'accueil pourraient remédier à un manque de main-d'œuvre immédiat et à moindre coût, les pays d'origine profiteraient des remises pécuniaires faites par les migrants à leurs familles et, enfin, ces derniers auraient accès à une source de revenus supérieure à celles qui sont disponibles dans leur pays. » (Bélanger et Candiz, 2014 p. 60).

Si des bienfaits économiques semblent incontestables (Bronsard, 2007), les politiques relatives à la migration temporaire ne sont toutefois pas exemptes de critiques dans la littérature scientifique. Certaines visent à dénoncer une forme d'institutionnalisation des inégalités entre les migrants, basée sur une distinction en matière de statut et de programmes dont ils dépendent. En effet, plusieurs auteurs (Le Ray, 2011 ; Morice, 2004 ; Pellerin, 2011) observent que les politiques de migration temporaire relèvent d'une vision utilitariste qui instaure de fait un régime migratoire à deux vitesses (Piché, 2009) distinguant d'un côté les migrants permanents, et de l'autre les migrants temporaires, dont les conditions de migration deviennent de plus en plus précaires. Les premiers peuvent ainsi légitimement viser une intégration dans le prolongement de leur migration et bénéficient de services dans cette optique (par exemple, des cours de langue), alors que les seconds ne peuvent prétendre à l'installation, et ce, même s'ils peuvent revenir d'une année sur l'autre. Cette absence de volonté politique d'intégration des travailleurs étrangers temporaires (Amar, et coll., 2009) se manifeste par l'absence de mesures d'aide juridique, d'aide sociale et d'instruction publique, notamment en matière de cours de langue (Centre de recherche en immigration, ethnicité et citoyenneté [CRIEC], 2012). En somme, la migration temporaire permettrait aux états qui s'en prévalent de tirer certains bénéfices économiques de la migration sans avoir à assumer les responsabilités sociales qui en découlent.

$\mathrm{Au}$ Canada, les travailleurs agricoles étrangers relèveraient de cette logique (CRIEC, 2012). Leur mobilité s'incarne dans deux programmes (PTAS et PTÉT-PS) rivalisant de flexibilité auprès des entreprises du secteur agro-industriel (Soussi, 2013).

1/ Le Programme de Travailleurs Agricoles Saisonniers (PTAS) : en 1966, le gouvernement fédéral du Canada instaure le PTAS avec la Jamaïque. Par la suite, ce programme est élargi à d'autres pays, notamment le Mexique en 1974 (Bronsard, 2007) qui est depuis devenu la principale source de main-d'oeuvre pour ce programme (Soussi, 2013). Bellerose et Yorn (2013) précisent que ce programme, mis en place au départ pour répondre de façon temporaire à une demande émanant des entrepreneurs face à une pénurie de main-d'œuvre dans le secteur agricole est depuis devenu un programme essentiel aux yeux des représentants agricoles. Le nombre de TÉT embauchés par l'intermédiaire du PTAS a presque triplé entre 2000 et 2009, passant de 16710 à 43028 travailleurs (Gravel et coll., 2014). C'est également le cas au Québec, où « en moins de dix ans, le nombre de Mexicains participant au PTAS a plus que triplé, évoluant de 797 travailleurs en 1996 à plus de 4300 en 2006, et ce, travaillant pour le compte de 325 employeurs » (Bronsard, 2007, p. 11).

2/ Le Programme des Travailleurs Étrangers Temporaires Peu Spécialisés (PTÉTPS) : en 2002, les autorités canadiennes mettent en place le PTÉT-PS pour soutenir la main d'œuvre étrangère dans les professions exigeant un niveau réduit de formation (Soussi, 2013). Ce programme ne se limite pas au secteur d'emploi agricole, mais à partir de 2002, il permet à ce dernier d'engager des travailleurs dans le pays de leur choix (Bellerose et Yorn, 2013). Ce faisant, le PTÉT-PS devient une voie supplémentaire de recrutement des 
travailleurs agricoles étrangers (Soussi, 2013) dans la mesure où les producteurs agricoles sont libres d'opter pour l'un ou l'autre de ces programmes, à condition qu'ils en respectent les exigences spécifiques (Bellerose et Yorn, 2013). En particulier, le contrat les lie aux travailleurs pour une durée maximale de huit mois dans le cadre du PTAS ou pour une durée maximale de douze mois dans le cadre du PTÉT-PS (Bronsard, 2007).

Au Québec, pour l'ensemble des deux programmes, le nombre de TÉT a connu une croissance d'environ $40 \%$ de 2000 à 2010, avec 30307 entrées en 2010 (Bélanger et Candiz, 2014), le secteur agricole contribuant en grande partie à cette augmentation. Sur le plan social, d'autres critiques s'attachent à dénoncer les situations défavorables vécues par les travailleurs temporaires peu qualifiés une fois arrivés dans les fermes (Bélanger et Candiz) à la fois en tant que groupes d'individus (échelle méso), et en tant qu'individus (échelle micro) particulièrement vulnérables et soumis à la précarité, ce qui fait l'objet de la section suivante.

\section{Contexte social des TÉT au Canada}

Bien que la littérature scientifique se soit moins intéressée au cas des TÉT qu'à d'autres catégories migratoires, les études disponibles ont mis en lumière plusieurs problématiques liées à leurs conditions de séjour, réputées difficiles, tant dans les fermes que dans le voisinage de celles-ci et ont souvent dépeint des situations préoccupantes.

En premier lieu, le statut des TÉT les place dans une situation de précarité et de vulnérabilité. En effet, ils sont admis sur le territoire canadien pour une durée limitée où ils sont contractuellement liés à leurs employeurs (Bellerose et Yorn, 2013 ; Bronsard, 2007) dont ils dépendent économiquement et juridiquement. Les TÉT sont alors décrits comme « une main-d'œuvre essentiellement masculine, prête à travailler au salaire minimum, disponible en tout temps » (Amar et coll., 2009 p. 6) et obligée de retourner vers sa communauté d'origine une fois le contrat de travail terminé. Bélanger et Candiz (2014) pointent d'ailleurs que la précarité et la vulnérabilité liées au statut de travailleur temporaire peu qualifié sont des constantes dans les travaux de recherche qui leur sont consacrés.

À la précarité découlant de leur statut migratoire et à la dépendance inscrite dans les rapports de travail qu'ils entretiennent avec leurs employeurs, s'ajoutent des conditions de travail souvent qualifiées de difficiles. Selon Soussi (2013), il est fréquent que les journées des travailleurs saisonniers dépassent les 15 heures pour des travaux faiblement rémunérés et socialement peu valorisés. Brem (2006) rappelle que les travailleurs agricoles sont parmi les plus exposés aux risques professionnels (pesticides, travail sous la chaleur extrême des serres, machines-outils, etc.), plus particulièrement encore lorsque la formation est inadéquate ou assurée dans une langue qu'ils ne comprennent pas.

L'éloignement géographique est un autre enjeu fréquemment rapporté. Il est causé, d'une part, par l'éloignement des TÉT de leur société d'origine (leurs familles en premier lieu) pour une durée considérable (Bellerose et Yorn, 2013), et d'autre part, par l'obligation de résider chez l'employeur, donc en zone rurale. Ce double éloignement est susceptible de générer chez les TÉT un sentiment d'isolement social, culturel et affectif grave (Amar et coll., 2009). Ainsi, Bélanger et Candiz (2014) relèvent que la notion d'invisibilité est omniprésente dans les entretiens qu'il a menés auprès de populations locales lorsque ces dernières évoquent leurs représentations au sujet des TÉT. Morice (2004), évoque pour sa part une formule, celle du « travail sans le travailleur », laquelle résume bien l'idée du peu d'espace d'existence sociale octroyé à ces groupes d'individus au-delà de leur simple 
fonction de travailleur. En somme, il semble que « les travailleurs étrangers sont des migrants idéaux, car ils répondent à un besoin en main-d'oeuvre sans reconfigurer l'ordre social, étant donné leur exclusion sociale, légale et spatiale » (Bélanger et Candiz, 2014, p. 61). Cet isolement social peut, dans certains cas, être accentué par des comportements discriminatoires et racistes de certains individus locaux à l'égard des TÉT (Preibisch, 2004), lesquels sont perçus comme culturellement et socialement différents (couleur de peau, statut de travailleur provenant d'un milieu pauvre ou encore venant « voler notre travail ») (Bellerose et Yorn, 2013 ; CRIEC, 2012).

\section{Contexte sociolinguistique des TÉT}

Sur le plan sociolinguistique, le fait de vivre quasi exclusivement dans les fermes et sur les terres agricoles limite considérablement les occasions d'interactions avec les individus locaux (Bélanger et Candiz, 2014 ; Bellerose et Yorn, 2013 ; Preibisch, 2004). Lorsqu'elles surviennent, elles sont contraintes par des limites linguistiques omniprésentes du fait que la plupart des TÉT ne parlent pas français, alors que la population locale ne maitrise habituellement pas la langue première des TÉT, qui correspond majoritairement à l'espagnol.

Cette barrière linguistique s'exprime en premier lieu au sein des fermes et explique pourquoi la relation professionnelle entre les TÉT et leurs employeurs ne peut pas se développer autant que voulue (Bronsard, 2007). Bien qu'une volonté de connaitre la langue de leurs interlocuteurs ait été relevée par Bellerose et Yorn (2013), du côté des TÉT comme du côté de leurs employeurs, la barrière linguistique prévaut dans la mesure où des cours de français ne sont pas prévus pour les TÉT, ni dans leur pays d'origine, ni une fois sur place. L'existence de rares cours de français au Québec est toutefois mentionnée par la Fondation des entreprises en recrutement de main-d'œuvre agricole étrangère (FERME), l'organisme chargé d'administrer les programmes PTAS et PTEP-PS relevant du secteur agricole au Québec.

La barrière linguistique se manifeste avec plus de force en dehors des fermes, lors des interactions limitées que les TÉT entretiennent avec la population locale autre que leurs employeurs. Brem (2006) ramène ces dernières principalement aux situations de transactions commerciales (achats, transactions bancaires), de soins de santé (pharmacie, clinique) ou de rares événements sociaux organisés par des organismes communautaires, syndicaux ou par des églises (Preibisch, 2004). Ainsi, les rares interactions que les travailleurs migrants agricoles développent avec la population locale restent principalement instrumentales (employeur, épicier, pharmacien ou banquier) et peu sociales, et ce, même s'ils reviennent chaque année dans une même ferme (Bellerose et Yorn, 2013). La barrière linguistique à laquelle sont confrontés les TÉT en dehors des fermes est mentionnée dans l'ensemble de leurs situations de vie. Bellerose et Yorn (2013) ont montré que la majorité des TÉT qu'il a interrogés ne sont pas accompagnés par des interprètes lors de leurs déplacements et doivent par conséquent se débrouiller individuellement en matière linguistique avec un bagage limité. Nombre d'entre eux témoignent ainsi de difficultés quand ils se rendent à la banque, à la pharmacie et à l'épicerie. Comme le résument Bélanger et Candiz (2014, p. 58) : «La barrière de la langue est constamment mentionnée pour expliquer l'impossibilité d'interagir. »

Ainsi, la barrière linguistique teinte l'ensemble de l'expérience des TÉT et amplifie la plupart des problématiques évoquées dans les études consacrées aux TÉT. Plus 
spécifiquement, dans le cas du Canada et du Québec, les problématiques linguistiques sont notamment présentes dans des travaux portant sur l'accès aux soins (Amar et coll., 2009) ; la santé et la sécurité au travail (Gravel et coll., 2014) les représentations sociales du point de vue des populations locales (Bélanger et Candiz, 2014); et les liens entre employeurs, TÉT et communautés d'accueil (Bellerose et Yorn, 2013 ; Preibisch, 2004). Or, bien qu'elle soit centrale et omniprésente dans la littérature consacrée aux TÉT, la problématique linguistique n'a pas été étudiée en tant qu'objet. Elle apparait davantage en filigrane d'autres objets d'étude, en tant que facteur explicatif. Par conséquent, l'objectif de notre étude est de mieux comprendre la situation sociolinguistique des TÉT lors de leur séjour dans les fermes du Québec.

\section{Éléments conceptuels}

Pour analyser la situation sociolinguistique des TÉT, nous nous appuyons sur le concept d'appropriation linguistique (Adami, 2012a ; Castellotti, 2012 ; Porquier, 1994) et évoquons spécifiquement, à l'intérieur de celle-ci, les principales voies sociolangagières d'acquisition en milieu social identifiées par Adami (2012b).

D'un point de vue terminologique, l'appropriation linguistique se définit comme un hyperonyme (Porquier, 1994) recouvrant à la fois les notions d'apprentissage (principalement en milieu de formation) et d'acquisition (principalement en milieu social ou milieu naturel) de la langue (Adami, 2012a). Elle regroupe ainsi l'ensemble des milieux, des modes (des plus formels aux plus informels) et des processus contribuant à l'élargissement des répertoires linguistico-culturels des individus (Castellotti, 2012).

Par le fait même d'être présents dans la société du pays d'accueil, les migrants se trouvent dans une situation d'immersion linguistique (Adami, 2012a, 2012b) où l'appropriation de la langue dominante peut se produire de prime abord en milieu social, source de multiples expositions et interactions linguistiques, et parfois en milieu de formation. En effet :

la plus grande part des acquis langagiers des migrants se structure au contact des natifs dans les multiples situations sociales de communication, et la formation, quand elle a lieu, n'est qu'un moment du long processus d'apprentissage de la langue dominante par les migrants (Adami, 2012b, p. 51).

L'appropriation linguistique est ainsi à même d'éclairer l'ensemble des trajectoires non linéaires et composites (Porquier, 1994) que peuvent emprunter les migrants lorsqu'il s'agit pour eux de s'approprier la langue dominante de leur milieu d'accueil. Dans le cas spécifique des TÉT, ces derniers ne semblent bénéficier que rarement d'un apprentissage formel du français, que ce soit dans leur pays d'origine ou lors de leur(s) séjour(s) au Québec. En revanche, une exposition au français semble davantage exister en milieu social, du fait de leur situation d'immersion (par exemple, au contact avec des employeurs, des organismes, des commerçants ou avec la population locale) et ce, de façon répétée dans le cadre de séjours successifs. En milieu social, Adami (2012b) précise que l'appropriation linguistique s'actualise selon deux modes complémentaires : principalement au travers de leurs interactions verbales (orales et écrites) avec des locuteurs de la langue (natifs ou non natifs) et complémentairement par le biais d'alteractions (exposition à des 
messages qui n'appellent pas de réponse). Bien que l'impact de ces dernières soit plus difficile à cerner, leur rôle peut se révéler essentiel, dans la mesure où elles conditionnent en partie la vie des TÉT lors de leur(s) séjour(s) au Québec.

Adami (2012b) identifie quatre voies sociolangagières majeures participant à l'appropriation du français en milieu social :

- le milieu de travail, où les pratiques de communication se révèlent particulièrement nombreuses (entre salariés, avec la direction et l'encadrement, avec les clients et partenaires de l'entreprise, par l'activité syndicale, par les écrits professionnels et par la formation), y compris dans les secteurs d'activité employant de la main-d'œuvre peu ou pas qualifiée, pour peu que le français y soit utilisé ;

- les relations transactionnelles en français, définies comme des relations de service dans lesquelles les interactants agissent dans des rôles spécialisés (entre clients et vendeurs, entre usagers et fonctionnaires ou d'autres acteurs d'institutions scolaires, d'associations, etc.) ;

- les relations interpersonnelles en français, constituées des échanges dans lesquels les interactants n'interviennent pas à titre spécialisé (échanges familiaux, amicaux, aléatoires et de routine) ;

- les alteractions en français, composées de messages lus ou entendus qui n'appellent pas de réponse (affiches, signalisation, annonces, presse, etc.).

Cette prise en compte des principales voies sociolangagières de l'appropriation linguistique rend possible une lecture opérationnelle en matière de situations spécifiques rencontrées ou non par les TÉT en situation d'immersion et en milieu social (aussi bien à l'échelle de la ferme qu'à l'extérieur de celle-ci), à défaut de bénéficier de cours de français. Elle permet ainsi de déterminer et de mettre en relation les occasions et les barrières linguistiques que rencontrent les TÉT dans leur quotidien en fonction de leurs compétences linguistiques. Toutefois, cette étude révèle que les TÉT font face à davantage de barrières que d'occasions linguistiques dans les voies sociolangagières auxquelles ils ont accès, ce qui explique pourquoi les barrières linguistiques ressortent de façon proéminente dans les résultats obtenus.

\section{Méthodologie}

Dans cette section, nous commençons par présenter brièvement les caractéristiques des participants. Nous exposons ensuite les outils de collecte de données, constitués d'un questionnaire et d'entrevues de groupe, avant de préciser l'analyse des données recueillies.

\section{Participants}

Notre étude s'est déroulée dans une ferme québécoise spécialisée dans la production de divers légumes et accueillant depuis plusieurs années des travailleurs immigrants saisonniers originaires du Mexique et du Guatémala. Vingt-quatre travailleurs immigrants saisonniers (hommes uniquement) hispanophones (14 Mexicains et 10 Guatémaltèques) représentaient l'essentiel des ouvriers agricoles présents à la ferme. Âgés de 22 à 46 ans, ils 
provenaient majoritairement de zones rurales $(66,7 \%)$ ou de zones semi-urbaines $(25 \%)$. Ils étaient en grande majorité mariés ( $71 \%)$ ou en union libre $(17 \%)$ et seulement l'un d'entre eux n'avait pas d'enfants à charge au moment de la collecte de données. Leur niveau d'éducation était relativement polarisé : 10 participants ont suivi une scolarité au primaire, alors que 13 autres ont poursuivi au secondaire. Un seul participant s'est rendu jusqu'au postsecondaire. Finalement, seuls trois participants étaient TÉT au Canada pour la première fois. À l'inverse, une majorité de participants avait une expérience de deux à quatre ans (15) en tant que TÉT, et de cinq à huit ans (4) ou de neuf ans et plus (2).

\section{Collecte de données}

La collecte des données a consisté en un questionnaire et trois entrevues de groupe auprès des 24 travailleurs immigrants saisonniers. Le questionnaire était centré sur les usages linguistiques des TÉT et sur l'autoreprésentation de leurs compétences linguistiques en espagnol (ou une autre langue d'origine que l'espagnol, pour le cas des TÉT autochtones), en français et en anglais. La première partie du questionnaire visait à mieux comprendre la distribution de ces langues en matière de situations (p. ex., à la ferme, à l'extérieur de la ferme : la banque, l'épicerie, etc.) et d'interlocuteurs (p. ex., l'employeur et le personnel administratif de la ferme, les collègues, etc.). La deuxième partie du questionnaire visait à considérer les besoins linguistiques des TÉT notamment dans l'optique d'explorer la pertinence et la possibilité de développer un cours de français pour cette population précise. Le questionnaire a été validé par le personnel de la ferme puis a été traduit en espagnol et mis en page de façon à soutenir au mieux la compréhension des participants, dont la compétence écrite était très variable (Figure 1). Finalement, la passation des questionnaires a été effectuée sur papier avec le soutien oral d'une locutrice parlant espagnol, afin que les questions soient lues oralement et que les participants puissent poser des questions si nécessaire. 


\section{Figure 1}

Extrait du questionnaire sur les usages technologiques des travailleurs immigrants saisonniers

13. Cuando está en su país de origen, ¿̇cuántas veces usa el equipo o las aplicaciones tecnológicas siguientes? (Señale la sección adecuada con una X.)

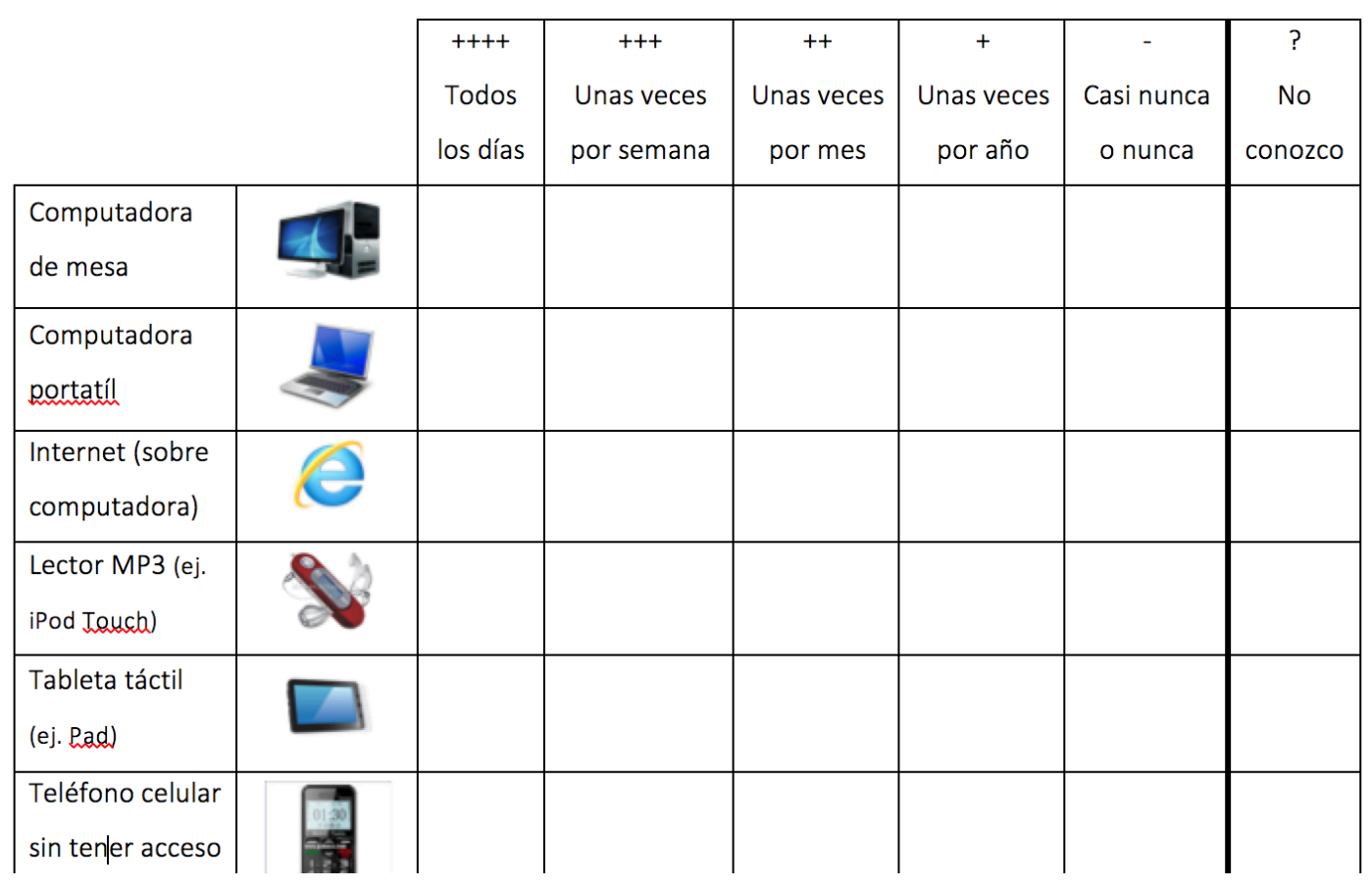

Le remplissage du questionnaire a été suivi par de courtes entrevues de groupe (20 à 25 min.) en raison des contraintes temporelles des TÉT. Le but des entrevues était de déterminer les logiques d'action et les raisons de la situation linguistique des TÉT, telle que décrite dans le questionnaire.

\section{Analyses de données}

Nous avons procédé à une analyse descriptive des réponses au questionnaire afin de dresser un portrait de la situation sociolinguistique des TÉT. Étant donné la taille de notre échantillon et la nature exploratoire de notre étude, nous avons essentiellement calculé des fréquences afin de déterminer les tendances principales.

Les entrevues ont été transcrites puis codées selon une analyse de contenu (L'Écuyer, 1990) à l'aide du logiciel QDAminer. Les catégories de code ayant émergé ont trait aux : connaissances linguistiques écrites et orales des participants dans les différentes langues ; situations linguistiques auxquelles ils sont confrontés ; types d'acteurs avec lesquels ils sont en contact ; relations qu'ils entretiennent avec les différents locuteurs ; relations que les autres locuteurs entretiennent avec eux ; difficultés linguistiques auxquelles ils sont confrontés; stratégies auxquelles ils font appel pour remédier à leurs difficultés linguistiques ; visées projetées quant à un apprentissage du français de base ; formes que pourrait prendre un cours de français leur étant consacré. 


\section{Résultats}

Dans une première section des résultats, nous dressons un portrait de la situation sociolinguistique des TÉT. Nous commençons par présenter les compétences linguistiques autorapportées des participants en espagnol, en anglais et en français qui s'avèrent limitées dans les trois langues. Nous relevons ensuite qu'ils disposent d'un nombre réduit de situations linguistiques à leur portée, situations dans lesquelles l'espagnol apparait comme leur principale langue d'usage. Nous indiquons ensuite les stratégies compensatoires qu'ils mettent en œuvre pour pallier leur difficile appropriation linguistique du français et le rôle ambigu que joue la société hôte à cet égard. Ces stratégies n'étant pas suffisantes pour réduire les bris de communication, nous concluons sur le fait que les participants vivent principalement une situation sociolinguistique caractérisée par des barrières linguistiques. À la suite de cette première section des résultats, nous nous concentrons, dans une seconde section, sur les besoins linguistiques exprimés par les TET afin de remédier à cette situation sociolinguistique. Nous commençons par présenter leur désir de mieux maitriser le français dans le double objectif de pouvoir agir et acquérir plus d'autonomie. Nous évoquons ensuite la solution qu'ils envisagent pour apprendre le français (celle-ci prendrait la forme de cours de français de base) et nous poursuivons en indiquant les modalités envisagées pour ces cours.

\section{Compétences linguistiques et situations linguistiques limitées}

Dans cette section, nous commençons par présenter les compétences linguistiques telles que les participants se les représentent, tant en espagnol qu'en français et en anglais, ainsi que les situations linguistiques dans lesquelles ils les exploitent.

\section{Compétences linguistiques limitées}

Un premier constat a trait à la perception de compétences linguistiques limitées par les participants, en français et en anglais, mais aussi en espagnol.

Concernant l'espagnol, la majorité d'entre eux rapporte un niveau intermédiaire en lecture ou en écriture (10 participants), voire débutant en lecture (4 participants) ou en écriture (3 participants), tandis qu'une minorité (4 participants) déclare un niveau avancé en lecture ou en écriture. Sur 14 supports de lecture suggérés dans leur langue maternelle (p. ex., journaux, pages Internet, documents religieux ou officiels...), la majorité rapporte que ce ne sont pas des usages qu'ils pratiquent, mis à part les messages texte (textos, SMS) pour un peu plus de la moitié d'entre eux (13 participants) et la lecture de journaux pour près de la moitié d'entre eux (11 participants).

En anglais, presque tous les participants indiquent avoir un niveau débutant pour les compétences de compréhension et de production à la fois orales et écrites, à de rares exceptions près. En effet, pour ces mêmes compétences, une minorité d'entre eux rapporte un niveau intermédiaire (entre un et trois participants selon la compétence évoquée), voire un niveau avancé pour un participant en lecture ou en écriture.

Finalement, en français, les tendances sont similaires puisque presque tous les participants déclarent avoir un niveau débutant pour les compétences de compréhension et de production à la fois orales et écrites. Seul un participant rapporte une compétence de niveau intermédiaire en compréhension et en production orales, ce qui s'explique par son 
statut professionnel au sein de la ferme : en tant que superviseur, il est tenu de rédiger des rapports pour l'administration de la ferme.

La figure ci-dessous (Figure 2) présente la distribution des compétences linguistiques autorapportées des participants en espagnol, en anglais et en français.

\section{Figure 2}

Distribution des compétences linguistiques autorapportées par les participants en espagnol, en anglais et en français.

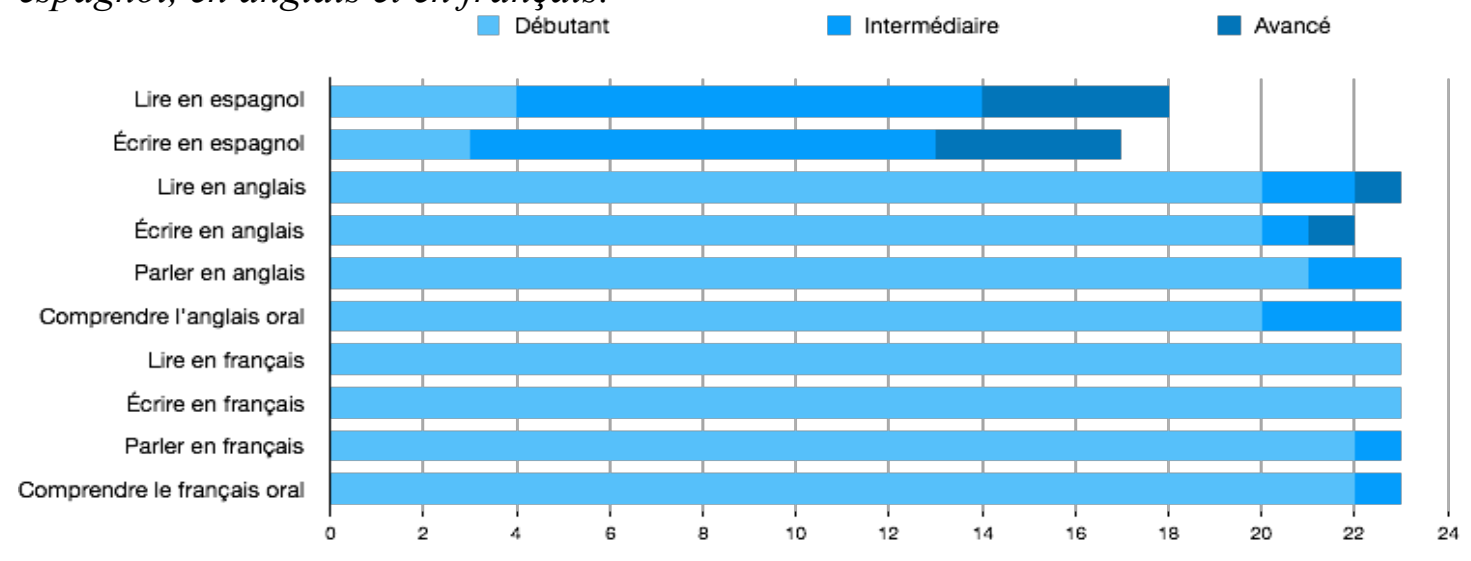

En résumé, les principales compétences linguistiques autorapportées par les participants se concentrent sur l'espagnol, notamment en ce qui a trait à la production et la compréhension orales. En revanche, le français semble être la langue la moins maitrisée par les participants, alors même qu'elle constitue la langue d'usage de la société hôte, donc la langue à laquelle ils sont le plus susceptible d'être exposés. La section suivante permet de mettre ces premiers résultats en perspective, en constatant que les participants ont peu d'occasions d'être exposés au français dans leur quotidien.

\section{Situations linguistiques limitées}

Au travers du sondage et des entrevues, les participants rapportent un nombre limité de situations linguistiques. Trois d'entre elles émergent de leur propos, dans lequel l'espagnol est la principale langue d'usage.

La situation linguistique la plus fréquemment rapportée (17 occurrences dans les entrevues) a trait au milieu professionnel, qui équivaut à la ferme où ils travaillent et où ils sont logés également. Les interactions ont majoritairement lieu en espagnol. En effet, selon les résultats du questionnaire, l'espagnol est la langue la plus souvent utilisée par les participants pour communiquer avec les autres TÉT, ainsi qu'avec les superviseurs (sauf pour un participant qui déclare utiliser le plus souvent le français avec ce type d'interlocuteurs) et avec l'administration de la ferme (sauf pour un participant qui déclare utiliser plus souvent le français et un autre l'anglais avec ce type d'interlocuteurs). Le français est donc peu utilisé, que ce soit à l'oral ou à l'écrit, sauf pour trois participants pour qui leur rôle de superviseur implique la rédaction de rapports.

La deuxième situation linguistique la plus fréquemment rapportée (14 occurrences dans les entrevues) concerne le milieu extra-professionnel (en dehors du site de la ferme). Bien qu'il y reste prépondérant, l'usage de l'espagnol apparait un peu moins exclusif qu'en milieu professionnel. En effet, selon les résultats du questionnaire, la langue déclarée 
comme étant la plus souvent utilisée est majoritairement l'espagnol. Cela se produit plus particulièrement dans le cadre de transactions médicales (pour 18 participants, contre une utilisation plus fréquente de l'anglais pour 4 participants ou du français pour 2 d'entre eux) ou de transactions bancaires (pour 17 participants, contre une utilisation plus fréquente de l'anglais pour 5 participants ou du français pour 2 d'entre eux) et de façon moins marquée dans le cadre de transactions avec les commerçants (pour 12 participants, contre une utilisation plus fréquente de l'anglais pour 6 participants ou du français pour 6 autres).

La dernière situation linguistique la plus récurrente des participants (17 occurrences dans les entrevues) a trait aux contacts qu'ils entretiennent avec la société d'origine et qui ne se situe pas à proprement parler au travail ni en dehors de celui-ci puisqu'elle est "déterritorialisée". Cette situation linguistique est réalisée quotidiennement en espagnol et revêt une charge affective considérable pour les participants. En effet, à la question de savoir s'ils communiquent avec des personnes de leur société d'origine, les participants répondent sans équivoque :

« c'est la chose la plus importante »

« essentiel»

« indispensable »

« oui parce qu'on a besoin de rester en contact avec nos familles » « avec un cellulaire on peut communiquer avec nos familles tous les jours »

En somme, l'espagnol est de loin la principale langue que les participants utilisent dans le nombre limité de situations linguistiques qu'ils ont à leur disposition. Le recours à l'anglais et au français est moindre et se produit éventuellement lors d'interactions en milieu extra-professionnel (deuxième situation linguistique), lequel se résume à trois types de transactions : bancaires, médicales et commerciales. Cette prévalence de l'espagnol en milieu francophone implique, pour les participants, la mise en œuvre de certaines stratégies compensatoires qui leur permettent de communiquer au mieux, ce qui fait l'objet de la section suivante.

\section{Stratégies compensatoires de communication}

Les participants rapportent quelques stratégies compensatoires visant à rendre possible la communication dans leur quotidien. En premier lieu, une petite minorité de participants indique recourir à une autre langue que l'espagnol, notamment l'anglais (dans 15 cas sur 72) et secondairement le français (dans 10 cas sur 72), lors de leurs interactions en milieu extra-professionnel (voir deuxième situation linguistique de la section Situations linguistiques limitées) :

« si on a appris un peu d'anglais et un peu de français, c'est plus facile de se faire comprendre. Ou même si tu sais un petit peu d'anglais et rien de français, parfois les gens parlent les deux et on peut communiquer en anglais » « dehors dans les magasins, il y a beaucoup de gens qui parlent anglais » 
Les résultats dont nous disposons ne nous permettent toutefois pas de comprendre quels sont les déclencheurs et les circonstances de ce passage de l'espagnol à une autre langue. À défaut de pouvoir recourir à l'anglais ou au français, l'ensemble des participants en est réduit à faire usage du non verbal et du paraverbal :

« beaucoup d'entre nous ici ne parlent pas français, nous ne savons pas quoi répondre, on n'utilise que des gestes » « si on doit communiquer en français, il nous arrive de ne rien comprendre, on communique seulement par des gestes » « seulement par des gestes tu dois te faire comprendre [dans un commerce]»

Deux autres stratégies, plus ponctuelles, ont également été relevées. Dans certains types d'interactions, notamment celles liées à des transactions médicales, la présence d'un interprète est nécessaire et permet alors aux participants de parler espagnol tout en assurant une communication de qualité :

« j'aimerais bien comprendre [le français] [...]. Lorsqu'on doit aller à l'hôpital, on pourrait se faire comprendre sans avoir besoin d'un interprète »)

Bien que marginale, une dernière stratégie rapportée par les participants consiste à utiliser des logiciels de traduction ou à choisir des interfaces en espagnol, par exemple pour l'ordinateur de bureau que la ferme met à leur disposition :

« notre ordinateur, on a besoin de changer pour l'espagnol pour pouvoir communiquer, pour savoir plus vite»

Face à ces stratégies compensatoires de communication, le milieu social joue un rôle ambigu, en faisant à la fois preuve d'adaptation et de marginalisation.

\section{Le rôle ambigu du milieu social}

Comment réagit le milieu social face à la condition linguistique des participants ? Cette section présente d'abord les initiatives d'adaptation qu'il met en place, tout en relevant certains indices de marginalisation des participants.

\section{Initiatives d'adaptation}

Les principales initiatives d'adaptation du milieu social à la condition linguistique des participants consistent à parler espagnol afin de faciliter la communication :

« le patron peut nous parler en utilisant un peu l'espagnol» « parfois on est seuls ou dans un magasin [...] Parfois, on trouve quelqu'un [parlant espagnol] » 
Cette adaptation est toutefois à géométrie variable dans la mesure où elle dépend de la présence de certains interlocuteurs et de leur compétence linguistique en espagnol. En outre, une majorité d'interlocuteurs francophones ne semblent pas parler espagnol de sorte que cette initiative reste restreinte dans son application :

« lorsqu'on a besoin de quelque chose, il y a personne, les gens ne parlent pratiquement pas en espagnol dans les magasins »

Une alternative aux interactions en espagnol consiste à traduire les pancartes et les consignes en espagnol, ce qui semble être le cas en milieu professionnel. Cette adaptation semble plus « autonomisante » pour les participants dans la mesure où elle ne nécessite pas la présence d'une tierce personne :

« dans cette ferme c'est bien parce qu'il y a beaucoup de choses qui sont en espagnol et en français [...] si on fait attention aux panneaux qui sont en français et en espagnol, tu sais de quoi il s'agit et tu peux bien comprendre »

Bien qu'elles favorisent peu l'autonomie linguistique des participants, ces initiatives ont le double mérite de prendre en compte leur condition linguistique particulière et d'impliquer les interlocuteurs francophones dans la manière d'y répondre. Toutefois, ces initiatives louables semblent contrecarrées par des indices de marginalisation du milieu social à l'égard des participants.

\section{Indices de marginalisation}

Les propos des participants font ressortir des logiques de marginalisation envers eux, dont la langue est l'un des marqueurs apparents. Ces logiques de marginalisation semblent majoritairement s'exprimer en milieu extra-professionnel et revêtir deux formes principales. La première consiste en des attitudes négatives de la part des interlocuteurs francophones, en matière de moquerie, de mépris ou d'ignorance :

« il m’est arrivé que quelqu'un se moque de nous par fait de ne pas pouvoir communiquer » « et en dehors du travail aussi, [...] pour faire des achats, $[\ldots]$ ils ne nous prennent pas en compte même si tu leur parle en français ou en espagnol, ils font semblant de ne pas comprendre »

La deuxième forme de marginalisation consiste à tirer profit de la précarité linguistique des participants pour les extorquer, notamment lors de transactions commerciales :

« ou peut-être lorsqu'on achète quelque chose elle coûte moins ou plus et nous ne comprenons pas »

« même une fois, un collègue est allé acheter une carte téléphonique de $20 \$$ et on lui a donné une de $30 \$$ pour son cellulaire. Puisqu'on ne comprend pas tu ne peux pas communiquer avec les autres » 
Ces indices de marginalisation sont moins rapportés en milieu professionnel. Les seules attitudes négatives répertoriées sur le site de la ferme sont relatives à la frustration de certains interlocuteurs francophones suite à des incompréhensions. En revanche, ces frustrations sont d'autant plus vivement ressenties par les participants qu'elles s'inscrivent dans des relations professionnelles hiérarchiques dont ils tiennent la position faible, ce qui réduit leur marge de négociation :

« il y a des fois que [nom d'un interlocuteur francophone] peut se gêner, il se désespère parce qu'on le comprend pas »

Bien que soulevé à plusieurs reprises par les participants, le thème de la marginalisation n'est pas approfondi et peu abordé frontalement, ce qui peut sans doute s'expliquer par le fait qu'il s'agit d'un thème délicat. À titre d'exemple, lorsque l'animateur a tenté d'approfondir le sujet en posant la question suivante : « voulez-vous donner votre opinion sur cela ? », la réponse unanime a été : «non, non ».

Entre compétences et situations linguistiques limitées, stratégies compensatoires limitées pour tenter d'y répondre et rôle ambigu du milieu social, la caractéristique la plus prégnante de la condition linguistique des participants semble être celle de barrières linguistiques.

\section{Les barrières linguistiques comme quotidien linguistique}

En tant que thème convergent et transversal des propos des participants, les barrières linguistiques semblent le mieux caractériser leur condition linguistique. Elles sont essentiellement dues à des difficultés à comprendre et à se faire comprendre en français, au travail comme en dehors du travail.

Au travail, ces barrières linguistiques sont particulièrement dommageables à la transmission des consignes relatives aux tâches à réaliser :

« c'est une grande difficulté [recevoir des ordres] si tu n'arrives pas à comprendre »

« par exemple les contremaitres sont toujours canadiens québécois, ils nous donnent des instructions sur un travail à faire, il y a des choses qu'on ne comprend pas »

À ces difficultés d'interaction s'ajoutent, bien que moins fréquemment, des difficultés d'alteraction, par exemple dans le cadre de la compréhension de documents écrits :

«j'ai vu aussi que les superviseurs nous donnent des formulaires à remplir concernant le travail et tout [...] ils sont en français et nous le comprenons pas »

Les mêmes barrières linguistiques se reproduisent en milieu extra-professionnel :

« arriver à comprendre les gens dehors, par exemple dans les magasins, dans le commerce, tout ça. Ils nous disent des choses et 
nous ne comprenons pas »

Plus précisément, les barrières linguistiques que les participants évoquent se concentrent en particulier autour du vocabulaire et de la prononciation :

« il y a des fois qu'on le comprend mais pas tout. Et comme dit mon collègue, on veut répondre à ce qu'on nous demande, mais on ne connaît pas les mots exacts » « surtout avec la prononciation. Si on ne prononce pas bien, ils ne te comprennent pas. C'est quelque chose surtout comme on vient de dire, commencer à connaître des mots et leur prononciation »

En somme, quelle que soit la situation de communication évoquée, les barrières à la communication s'expriment d'abord dans des difficultés de compréhension. Lorsque cette difficulté est surmontée, c'est la possibilité de poursuivre l'échange qui devient problématique. Cette situation déficitaire est rapportée comme frustrante pour tous les participants et ces derniers se déclarent affectés par le fait de ne pas comprendre (11 occurrences dans les entrevues) et de ne pas se faire comprendre ( 8 occurrences dans les entrevues).

Pour dépasser les barrières linguistiques qui entravent leur quotidien linguistique, les participants évoquent régulièrement l'apprentissage du français. Cependant, cette solution n'est pas à leur portée de sorte qu'elle constitue un besoin exprimé, ce qui fait l'objet de la prochaine partie de cet article.

\section{Un désir déclaré de mieux maitriser le français}

Selon leurs déclarations au sondage, les participants manifestent quasi unanimement un fort intérêt à l'idée de perfectionner leurs compétences en français (23 sur 24 participants). Plus précisément, 11 d'entre eux déclarent que perfectionner leurs connaissances en français leur serait très utile et huit autres, que cela leur semble indispensable.

La figure ci-dessous (Figure 3) présente le niveau d'intérêt rapporté par les participants quant à un perfectionnement de leurs connaissances du français. 


\section{Figure 3}

Niveau d'intérêt rapporté par les participants quant à un perfectionnement de leurs connaissances du français

Pour vous, le perfectionnement de vos connaissances en français est...

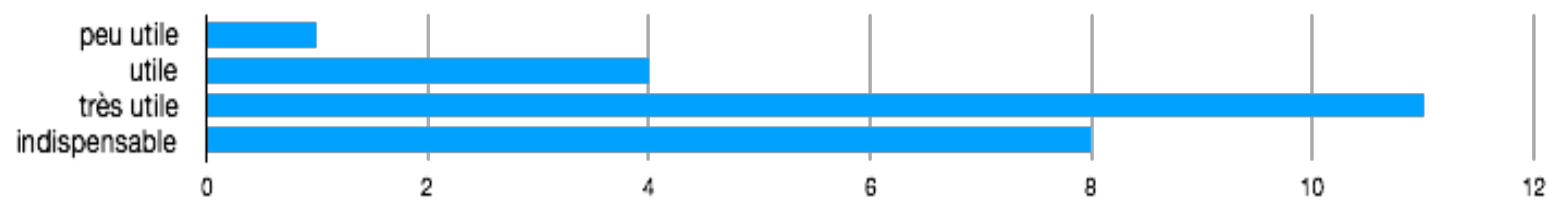

Les entrevues permettent de confirmer cet intérêt pour l'apprentissage du français :

« cela serait indispensable pour comprendre et réaliser de meilleure façon mon travail ici $[\ldots]$ comprendre les gens dehors $[\ldots]$ pour vivre mieux ici » « ici c'est le français, la langue de base c'est le français [...] ici la priorité est d'apprendre le français »

« c'est de base ici au Québec, pour se débrouiller [...] car c'est la première langue parlée ici alors, comme tu habites ici, tu dois essayer de parler français »

Les raisons pour lesquelles les participants expriment le besoin d'apprendre le français sont de natures diverses, que nous regroupons en deux catégories : la possibilité de communiquer pour agir, d'une part; et la possibilité d'acquérir plus d'autonomie, d'autre part.

\section{Communiquer pour agir}

Le français est vu comme la possibilité pour les participants de communiquer pour agir adéquatement. Plus précisément, les participants souhaitent en premier lieu être en mesure de comprendre le français (31 occurrences dans les entrevues), tant en milieu professionnel qu'extra-professionnel:

« ce serait indispensable pour comprendre et réaliser de meilleure façon mon travail ici »

« des fois, à cause d'une maladie, à la pharmacie, on a alors besoin de communiquer. C'est vraiment fondamental de comprendre »

En second lieu, les participants rapportent souhaiter être capables de s'exprimer en français (26 occurrences dans les entrevues), ce qui recouvre des notions différentes comme pouvoir parler (16 occurrences), pouvoir écrire ( 7 occurrences), se faire comprendre ( 2 occurrences) ou encore, remplir des formulaires (1 occurrence) :

« comprendre et parler aussi [...] lorsque tu essaies d'expliquer quelque chose à ton patron ou à n'importe qui, si tu ne parles pas, tu n'arriveras pas à communiquer » 


\section{Communiquer pour s'emanciper}

Outre cette vision pragmatique du français, certains participants rapportent que l'apprentissage du français leur permettrait de gagner en autonomie, notamment sur le plan du travail, mais aussi dans d'autres situations où la qualité de la communication peut être décisive (p. ex., lors de transactions médicales) :

« j’aimerais le comprendre bien surtout aussi, au travail, parce que parfois si le contremaitre ou notre superviseur est occupé, nous n'avons pas besoin de l'utiliser » « à l'hôpital, on pourrait se faire comprendre avec les gens sans avoir besoin d'un interprète » « surtout pour pouvoir nous débrouiller au travail avec les gens qui travaillent dans l'équipe »

Plus globalement, ce gain d'autonomie linguistique contribuerait à accroitre leur qualité de vie :

« il serait nécessaire de comprendre et savoir lire aussi pour vivre mieux ici, durant le temps qu'on sera ici, pour vivre mieux. Pour tout ça, j'aimerais savoir le français » « notre séjour ici serait mieux et la vie serait encore mieux pour nous si l'on parle français »

\section{Une solution envisagée : des cours de français de base}

Dans la perspective de mieux fonctionner en français, d'accéder à une meilleure communication et de mieux vivre, les participants évoquent l'apprentissage du français sous la forme d'un cours de français de base (11 occurrences).

\section{Des cours avec une dominante orale}

D'après le questionnaire, si des cours de français étaient offerts, ils devraient privilégier la compréhension et la production orales en milieu professionnel et extraprofessionnel $(n=11)$. Toutefois, plusieurs participants souhaitent développer ces compétences exclusivement en lien avec le milieu extra-professionnel $(n=8)$ ou, secondairement, avec le milieu professionnel $(n=5)$.

La figure ci-dessous (Figure 4) présente l'adéquation d'un cours de français par rapport aux besoins linguistiques des participants. 
Figure 4

Adéquation d'un cours de français par rapport aux besoins linguistiques des participants

Oral : pour comprendre et parler $\quad$ Ecrit : pour lire et écrire

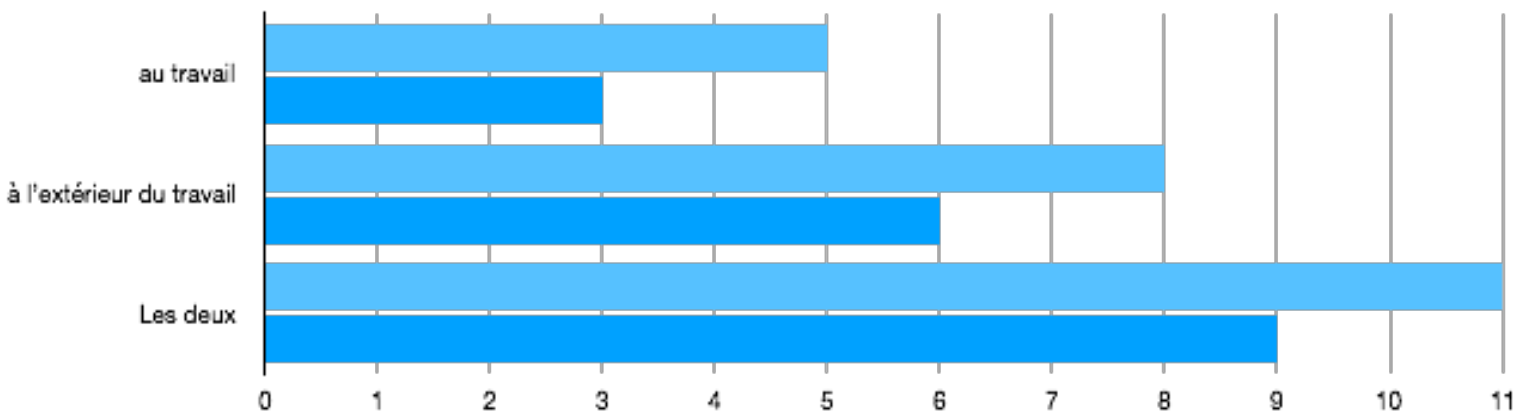

Des cours de groupe avec un enseignant en présentiel

Cette préférence pour l'oral est également perceptible lors des entrevues de groupe et se double d'une préférence pour une formule de groupe avec un enseignant en présentiel, ce qui permettrait de privilégier les interactions orales :

« en groupe, parce qu'on travaillerait ensemble »

«l'enseignant pourrait nous poser des questions et on pourrait en discuter mieux ensemble en groupe »

Cette formule semble privilégiée également parce que les participants ne se perçoivent pas comme étant capables d'apprendre de façon autonome, y compris en ayant recours aux technologies :

« c'est mieux [en salle de classe] parce qu'en ligne, honnêtement, parfois là, nous ne mettons pas la même attention, ni le même intérêt, ni le même désir, parce qu'on le néglige puisque c'est fatiguant »

« parce qu'en ligne, on ne le fera pas »

Cela semble conforme avec les réponses données lors du sondage où, quasi unanimement, ils envisageaient que les cours soient donnés par un enseignant en salle de classe. Un seul participant étant favorable à l'ajout de temps de formation à distance à ce cours en présentiel.

\section{Des cours conciliables avec leur vie de travailleur}

Enfin, selon le sondage, la durée de cours la plus adéquate serait de 60 minutes deux fois par semaine $(n=13)$, voire une fois par semaine $(n=6)$, suivie par un cours hebdomadaire de 90 minutes. Ce cours aurait lieu idéalement en fin de semaine pour une majorité de participants $(n=21)$, et plus particulièrement le soir en fin de semaine $(n=14)$.

En résumé, pour favoriser la participation du plus grand nombre, il semblerait que la forme de cours idéale selon ces déclarations soit celle d'un cours (voire deux) en salle de 
classe avec un enseignant, en soirée et en fin de semaine et d'une durée minimale d'une heure chacun.

\section{Discussion et conclusion}

Nous avons commencé par établir un portrait des compétences linguistiques autorapportées des TÉT en espagnol, en anglais et en français. Celui-ci s'avère limité dans les trois langues. Leurs compétences linguistiques se concentrent sur l'espagnol, notamment en ce qui a trait à la production et la compréhension orales. Le français semble être la langue la moins maitrisée par les participants, alors même qu'elle constitue la langue d'usage de la société hôte, donc la langue à laquelle ils sont le plus susceptible d'être exposés. Nous avons poursuivi en établissant un portrait des situations linguistiques à leur disposition au quotidien. Ces situations apparaissent limitées en nombre et se déroulent majoritairement en espagnol, offrant une faible exposition au français et par conséquent, un potentiel limité pour leur appropriation linguistique de la langue dominante. En effet, ces résultats indiquent en particulier que les quatre voies sociolangagières majeures participant à l'appropriation linguistique du français en milieu social identifiées par Adami (2012b) (milieu de travail, relations transactionnelles, relations interpersonnelles et alteractions) apparaissent particulièrement peu productives lors de leur séjour au Québec. De plus, les alteractions relevées sont peu nombreuses et lorsqu'elles se produisent à l'occasion de la consultation de formulaires en français au travail, ne semblent pas bénéficier à l'appropriation linguistique des participants dans la mesure où ces derniers disposent, selon leur propre perception, de faibles compétences en français, en particulier à l'écrit.

Nous nous sommes ensuite penchés sur les stratégies compensatoires que les TÉT mobilisent pour communiquer. Compte tenu de leurs compétences linguistiques limitées en français et en anglais, peu de stratégies sont à leur portée. De plus, au regard de la condition linguistique des TÉT, il apparait que le milieu social joue un rôle ambigu. Il fait preuve de certaines initiatives d'adaptation au travers de recours à l'espagnol qui se retrouvent davantage en milieu de travail. Bien que louables, ces initiatives se produisent en espagnol, ce qui ne participe pas à l'appropriation linguistique du français potentiellement attendue $\mathrm{du}$ fait de la situation d'immersion des participants. Ces initiatives semblent toutefois contrecarrées par des indices de marginalisation à l'égard des TÉT, qui sont davantage relevés en milieu extra-professionnel. Leur quotidien linguistique se caractérise donc par un ensemble de barrières linguistiques. Ces contraintes vécues dans les fermes et en milieu extra-professionnel affectent le séjour au Québec de tous les participants.

Compte tenu de compétences et de situations linguistiques limitées, de stratégies compensatoires limitées et du rôle ambigu joué par la société hôte, l'appropriation linguistique ne semble pas en mesure de se produire, malgré l'apparente situation d'immersion linguistique. Il est possible de penser que cette situation défavorable les distingue alors d'autres types de migrants (p. ex., les travailleurs qualifiés) qui, s'ils disposent de compétences linguistiques tout aussi limitées à leur arrivée, peuvent pour leur part bénéficier de situations linguistiques nettement plus riches et variées en situation d'immersion linguistique, favorisant leur appropriation du français. L'existence d'un régime migratoire à deux vitesses, basé sur une inégalité de statut (Le Ray, 2011 ; Morice, 2004 ; Pellerin, 2011 ; Piché, 2009) se reflèterait donc également dans une situation sociolinguistique inégalitaire au sein de laquelle l'immersion ne se traduit pas chez les TÉT 
en appropriation linguistique, contrairement aux migrants qui s'installent durablement dans la société hôte.

Nous nous sommes enfin tournés vers les solutions que les TÉT envisageaient pour faire face aux barrières linguistiques qui entravent leur quotidien linguistique. La solution privilégiée de façon quasi unanime est celle d'une meilleure maitrise du français. Ils expriment ce besoin dans la double perspective de communiquer et d'agir adéquatement au travail et dans la société, d'une part, et de communiquer pour s'émanciper et vivre mieux lors de leur(s) séjour(s) au Québec, d'autre part. Dans ce sens, les participants envisagent des cours de français de base sous la forme de cours de groupe avec un enseignant en présentiel. Ces cours seraient davantage orientés vers l'oral et les besoins en dehors du travail à des moments conciliables avec leur vie de travailleur.

Les cours de français apparaissent d'autant plus essentiels pour l'appropriation linguistique des TÉT que cette dernière ne semble pas se produire de manière informelle. Pour impulser cette appropriation linguistique, les TÉT doivent alors pouvoir bénéficier de ressources formelles et en premier lieu, de cours de français. Bellerose et Yorn (2013) ont d'ailleurs montré que cette solution recueille un intérêt de la part de certains employeurs du secteur pour lesquels la maitrise d'un français adapté permettrait une plus grande sécurité dans les fermes et une plus grande autonomie dans la prise en main de tâches qu'ils ne peuvent confier aux TÉT, faute d'une intercompréhension suffisante. Cette solution présenterait l'avantage d'améliorer la situation des TÉT lors de leurs séjours tout en améliorant celle de leurs employeurs, et ce, de façon durable, puisque les travailleurs reviennent souvent d'une année sur l'autre. Cependant, il convient de rappeler que mis à part de rares initiatives de formation des TÉT au français suggérées par FERME, celles-ci ne sont pas institutionnalisées (CRIEC, 2012). Compte tenu de l'ampleur du phénomène en matière de nombre de travailleurs impliqués annuellement et de l'enjeu que représentent les TÉT pour l'économie locale et la prospérité du Québec, des responsabilités semblent à prendre d'un point de vue politique.

Cette étude demeure fortement contextualisée en raison des spécificités de la population des TÉT, des programmes gouvernementaux et des relations avec la société locale. Elle est avant tout exploratoire. Dans sa foulée, il serait bon de préciser les aspects didactiques spécifiques qui conviendraient à une telle offre de formation, notamment en termes de modalités et d'approches didactiques.

D'un point de vue méthodologique, il est à signaler que l'étude des TÉT est délicate à opérationnaliser. Cette population est relativement peu accessible pour plusieurs raisons: 1/ sur le plan géographique, les TÉT résident dans des zones rurales parfois éloignées des universités; 2/ sur le plan organisationnel, ils ont de longues journées de travail, ce qui implique qu'ils ont peu de disponibilité quotidienne et qu'ils sont fatigués, ce qui est susceptible de limiter le temps et la qualité de la collecte de données; 3/ sur le plan linguistique, les entrevues impliquent nécessairement la présence d'un interprète, ce qui réduit la fluidité et la précision des échanges entre les chercheurs et les participants. En outre, le questionnaire doit également faire l'objet d'une traduction, d'une simplification et d'ajout d'aides visuelles (voir figure 1) afin de maximiser la compréhension des participants dont la compétence écrite, même en espagnol, est variable (voir section 5.5.1). Il s'agit de contraintes méthodologiques qui ont nécessairement teinté la collecte et l'analyse des données de la présente étude. Bien que nous en ayons conscience, ces contraintes nous semblent un mal nécessaire pour réaliser des études auprès des TÉT et, à ce titre, elles en sont partie prenante. 
La correspondance devrait être adressée à Simon Collin.

Courriel : collin.simon@uqam.ca

\section{Références}

Adami, H. (2012a). La formation linguistique des migrants adultes. Savoirs, (2), 9-44.

Adami, H. (2012b). Aspects sociolangagiers de l'acquisition d'une langue étrangère en milieu social. Dans Adami, H. et Leclercq, V. (dir.), Les migrants face aux langues des pays d'accueil : acquisition en milieu naturel et formation (pp. 51-87). Presses Universitaires du Septentrion.

Amar, M., Roberge, G., LaRue, A., Gélineau, L. et Leanza, Y. (2009). Les travailleurs agricoles migrants mexicains et guatémaltèques de l'Île-d'Orléans : Portrait des besoins de santé, de l'accessibilité et des trajectoires d'utilisation des services de santé (rapport de recherche-évaluation). Centre de santé et des services sociaux de la Vieille-Capitale.

Bélanger, D. et Candiz, G. (2014). Essentiels, fiables et invisibles : Les travailleurs agricoles migrants latino-américains au Québec vus par la population locale. Canadian Ethnic Studies, 46(1), 45-66.

Bellerose, J. et Yorn, C. (2013). Les travailleurs migrants sur les fermes du Québec (rapport de recherche). Centre d'innovation sociale en agriculture.

Brem, M. (2006). Les travailleurs migrants au Canada: une revue du Programme des travailleurs saisonniers agricoles du Canada (dossiers de politiques). Institut NordSud.

Bronsard, K. (2007). Main-d'œuvre mexicaine sur les terres agricoles québécoises : entre mythe et réalité [thèse de maitrise, Université Laval, Québec]. http://hdl.handle.net/20.500.11794/19718

Castellotti, V. (2012). Recherches qualitatives : épistémologie, écriture, interprétations en didactique des langues. Dans Goï, Cécile (dir.), Quelles recherches qualitatives en sciences humaines? Approches interdisciplinaires de la diversité (pp. 29-44). L'harmattan.

Centre de recherche en immigration, ethnicité et citoyenneté (CRIEC). (2012). Bulletin de l'Observatoire international sur le racisme et les discriminations, 8(1), 1-20.

Gravel, S., Villanueva, F., Bernstein, S., Hanley, J., Crespo, D. et Ostiguy, E. (2014). Les mesures de santé et sécurité au travail auprès des travailleurs étrangers temporaires dans les entreprises saisonnières. Perspectives interdisciplinaires sur le travail et la santé, 16(2).

L'Écuyer, R. (1990). Méthodologie de l'analyse développementale de contenu. Méthode GPS et Concept de soi. Presses de l'Université du Québec.

Le Ray, M. (2011). Migration temporaire, injustices durables. Le cas des travailleuses et travailleurs étrangers « temporaires » au Canada. Vivre ensemble, 1-13.

Morice, A. (2004). Le travail sans le travailleur. Plein droit, 61(2), 2-7.

Pellerin, H. (2011). De la migration à la mobilité : changement de paradigme dans la gestion migratoire. Le cas du Canada. Revue européenne des migrations internationales, 27(2), 57-75.

Piché, V. (2009). Les travailleurs migrants, nouveaux non-citoyens du monde. Possibles, $32(3-4), 41-51$. 
Porquier, R. (1994). Communication exolingue et contextes d'appropriation. Bulletin VALSASLA, 59, 159-169.

Preibisch, K. L. (2004). Migrant agricultural workers and processes of social inclusion in rural Canada: Encuentros and desencuentros. Canadian journal of Latin American and Caribbean studies, 29(57-58), 203-239.

Soussi, S. A. (2013). Les flux du travail migrant temporaire et la précarisation de l'emploi: une nouvelle figure de la division internationale du travail?. Revue multidisciplinaire sur l'emploi, le syndicalisme et le travail, 8(2), 145-170. 